\title{
Pixel-Based Image Processing for CIE Standard Sky Classification through ANN
}

\author{
D. Granados-López $\mathbb{D}^{1},{ }^{1}$ A. García-Rodríguez ${ }^{1}{ }^{1}{ }^{1}$ S. García-Rodríguez $(\mathbb{D})^{1}$ \\ A. Suárez-García $\mathbb{D}^{1},{ }^{1,2}$ M. Díez-Mediavilla $\mathbb{D}^{1},{ }^{1}$ and C. Alonso-Tristán $\mathbb{D}^{1}$ \\ ${ }^{1}$ Research Group Solar and Wind Feasibility Technologies (SWIFT), \\ Electromechanical Engineering Department, Escuela Politécnica Superior, 09006 Burgos, Spain \\ ${ }^{2}$ Centro Universitario de la Defensa, Escuela Naval Militar de Marín, Pontevedra 36920, Marin, Spain
}

Correspondence should be addressed to C. Alonso-Tristán; catristan@ubu.es

Received 9 September 2021; Accepted 26 November 2021; Published 20 December 2021

Academic Editor: Lingzhong Guo

Copyright (c) 2021 D. Granados-López et al. This is an open access article distributed under the Creative Commons Attribution License, which permits unrestricted use, distribution, and reproduction in any medium, provided the original work is properly cited.

\begin{abstract}
Digital sky images are studied for the definition of sky conditions in accordance with the CIE Standard General Sky Guide. Likewise, adequate image-processing methods are analyzed that highlight key image information, prior to the application of Artificial Neural Network classification algorithms. Twenty-two image-processing methods are reviewed and applied to a broad and unbiased dataset of 1500 sky images recorded in Burgos, Spain, over an extensive experimental campaign. The dataset comprises one hundred images of each CIE standard sky type, previously classified from simultaneous sky scanner data. Color spaces, spectral features, and texture filters image-processing methods are applied. While the use of the traditional RGB color space for image-processing yielded good results (ANN accuracy equal to 86.6\%), other color spaces, such as Hue Saturation Value (HSV), which may be more appropriate, increased the accuracy of their global classifications. The use of either the green or the blue monochromatic channels improved sky classification, both for the fifteen CIE standard sky types and for simpler classification into clear, partial, and overcast conditions. The main conclusion was that specific image-processing methods could improve ANN-algorithm accuracy, depending on the image information required for the classification problem.
\end{abstract}

\section{Introduction}

Sky conditions are crucial factors when assessing daylighting levels and solar-energy output. The sky is generally classified on the basis of cloud presence into three categories: cloudless, partially cloudy, and overcast. Many models for the calculation of global, direct, and diffuse irradiation and illumination were defined for different sky types based on the values of several climatic parameters [1]. In 2003, the Commission Internationale de L'Éclairage (CIE) adopted the set of 15 standard sky classifications proposed by Kittler et al., in 1998, categorized under 3 sky types, clear, partial, and overcast, each of five grades [2]. These CIE standard skies that classify a general spectrum of homogeneous skies throughout the world were standardized in ISO 15469: 2004(E)/CIE S 011/E:2003 [3] for the purpose of evaluating indoor visual comfort within buildings [4], solar irradiance calculations [5], and energy efficiency improvements to lighting [6], among other applications.

The CIE standard sky classification is based on taking luminance measurements [7] of diffuse luminance angular distribution in the sky vault. Skies within a CIE category have approximately the same well-defined sky luminance and solar radiance patterns.

Devices called sky scanners are used to measure sky luminance patterns. According to the CIE Guide [8], a reliable commercial sky scanner measures luminance from 145 patches of sky hemisphere. However, various alternative procedures have been developed for CIE standard sky classification [9], due to the scarcity of sky scanners available to gather sky luminance data at ground meteorological stations. In this task, Supervised Machine Learning (SML) 
procedures are proposed as effective tools for sky classification, based on accessible meteorological indices [10] such as decision trees (DTs) [11], Support Vector Machines (SVMs) [12], and Artificial Neural Networks (ANNs) [13-15].

Over recent years, interest has been expressed in calibrated sky luminance maps for sky classification and cloud detection [16-19]. A digital camera equipped with a fisheye lens can map at a higher resolution than commercial sky scanners and High Dynamic Range (HDR) images can capture the full sky luminance range [20].

There are also novel image-processing methods that can help to overcome misclassification due to cloud cover. While some studies have had their focus placed on color space, the focus of others has been on the modification and combination of the original monochromatic channels, known as the spectral features. A third alternative, texture filters, adjusts the gray pixel image patterns [21].

The RGB (red, green, and blue) chromaticity color model, a basic standard for computer images, has spectral features that may be adapted to cloud detection (CD) [22]. Shorter sunlight spectrum wavelengths will scatter due to atmospheric particles, giving the sky background a blue appearance [23] where the chromaticity component is mainly blue rather than red. Clouds appear white due to the uniform scattering of visible-light wavelengths, indicating similar amounts of red and blue components. Other models successfully applied to CD include Removal Atmospheric Scattering (RAS) [24], Red-Blue Ratio (RBR) [21], Red-Blue Difference (RBD) [25], and Normalized Red-Blue Ratio (NRBR) [17].

Some strategies have been aimed at adapting the image to the color perception of the human eye. Hue Saturation Value (HSV) [17], Red-difference Chroma (YCbCr) [18], and Intensity Hue Saturation (HIS) [26], among other color spaces, have recently demonstrated their efficacy for CD.

In addition to color space and spectral features, texture procedures use the gray distribution of pixels and their spatial neighborhood to identify objects and regions. These procedures have been shown to be very effective for cloud detection [27], medical images classification [28], and traffic analysis [29]. Gray Level Cooccurrence Matrix (GLCM), Local Range (LR), local Standard Deviation (STD), and local Entropy Matrix (EM) are texture filter procedures that statistically process the textures of images for their classification.

Image processing based on spectral, texture, and color spaces offers various perspectives of the same image. Their combination for image analysis can produce successful applications such as mapping [30] and aerial photographic classification [31]. In this paper, the recently proposed alternatives to the RGB color model are reviewed and compared for the improvement of image-processing methods applied to cloud detection and sky classification using Artificial Neural Network (ANN) algorithms. In some cases, preliminary image processing significatively improved the accuracy of the ANN used to classify the same image dataset. The methods that reduce misclassification will be identified from a detailed study, in which both the CIE standard sky classification (15 types) and the reduced classification of three categories (clear, partial, and overcast sky conditions) were all considered.

The paper will be structured as follows. A complete comparison between several image-processing methods for CIE standard sky classification though ANNs will be presented in Section 2. In Section 3, the acquisition and processing of the experimental data will be described. In Section 4 , the fit of the results of the ANN models with actual sky conditions will be verified. The results of the classification algorithms will be discussed in Section 5 and, finally, succinct conclusions on the most efficient image-processing methods will be presented in Section 6 .

\section{Review of Image-Processing Methods for Cloud Detection}

Table 1 summarizes the main characteristics of twenty-two pixel image-processing methods that were reviewed and tested in this study and classified in terms of color space, spectral, and texture features. A complete description of all the image-processing methods will be completed in this section.

2.1. Color Spaces. The RGB color space uses one channel for each of the primary colors: blue, red, and green. Implemented directly in machine learning or with previous processing, this color space will yield spectral features. The primary colors, subchannels $\mathrm{R}, \mathrm{G}$, and $\mathrm{B}$, build up a monochromatic image. A grayscale (GS) image is created when only pixel intensity is recorded. As previously mentioned, the HSV space is modelled on visual human perception, which classifies objects in terms of their luminous intensity (brightness or value) and chromaticity. The chromaticity has two independent parameters, hue and saturation. Hue is the pure color that varies from red to magenta (listed as red, yellow, green, cyan, blue, and magenta). The saturation describes the dilution of a pure color in white $(0=$ white; $1=$ pure color). The hue, saturation, and value channels can also be independently used. Clouds are mostly perceived on a grayscale, due to interactions between sunlight and the atmosphere, so different cloud cover can be analyzed through the saturation channel. This color space has proved itself to be highly effective for sky classification into three categories: blue sky, cloudy sky, and sunset sky [35].

2.2. Spectral Features Based on the RGB Model. Unlike the direct implementation of the RGB model, a spectral feature describes the change of tone and color in an image. Its capability of detecting dark clouds from high and transparent cirrus clouds has been demonstrated [25]. The RAS channel was proposed to distinguish atmospheric scatter from atmospheric background light [24]. The RAS channel is obtained from a linear combination of the panchromatic channel $(\mathrm{Y})$, the bright channel $(\mathrm{L})$, and the dark channel (D), defined in Table 1. Channels Y, L, and D can also be independently applied. 
TABLE 1: Summary of pixel image-processing methods.

\begin{tabular}{|c|c|c|c|c|}
\hline Type & Name & Purpose & Formulation & Ref. \\
\hline \multirow{5}{*}{ Color space } & RGB & Image visualization based on primary colors & $\begin{array}{l}\text { R: red channel } \\
\text { G: green channel } \\
\text { B: blue channel }\end{array}$ & {$[31]$} \\
\hline & $\begin{array}{l}\text { Subchannels R, G, } \\
\text { and B }\end{array}$ & \multicolumn{2}{|c|}{ R: red channel; G: green channel; B: blue channel } & \\
\hline & GS & $\begin{array}{c}\text { Grayscale intensity image (calculated from } \\
\text { the RGB image) }\end{array}$ & rgb2gray ${ }^{1}$ & [32] \\
\hline & HSV & $\begin{array}{l}\text { Image visualization based on the perceptions } \\
\text { of the human eye }\end{array}$ & $\begin{array}{l}\text { H: hue channel } \\
\text { S: saturation channel } \\
\text { V: value channel }\end{array}$ & [16] \\
\hline & $\begin{array}{l}\text { Subchannels H, S, } \\
\text { and V }\end{array}$ & \multicolumn{2}{|c|}{ H: hue channel; S: saturation channel; V: value channel } & \\
\hline \multirow{8}{*}{$\begin{array}{l}\text { Spectral } \\
\text { feature }\end{array}$} & RAS & $\begin{array}{l}\text { Image processing for removing atmospheric } \\
\text { scattering }\end{array}$ & $\begin{array}{c}R A S=Y-(L-D) \\
L=\max (R, G, B) \text { bright } \\
D=\min (R, G, B) \text { dark of the channel } \\
Y=0.299 \cdot R+0.587 \cdot G+0.114 \cdot B\end{array}$ & {$[21,24]$} \\
\hline & $\begin{array}{l}\text { Subchannels Y, L, } \\
\text { and D }\end{array}$ & \multicolumn{2}{|c|}{ Y: panchromatic channel; L: bright channel; D: dark channel } & \\
\hline & RBR & $\begin{array}{l}\text { Background due to atmospheric scattering } \\
\text { (Red-Blue Ratio). }\end{array}$ & $\mathrm{RBR}=\mathrm{R} / \mathrm{B}$ & {$[24]$} \\
\hline & $\mathrm{RBD}$ & Difference between red and blue channels & $\mathrm{RBD}=\mathrm{R}-\mathrm{B}$ & [33] \\
\hline & NRBR & $\begin{array}{l}\text { Blueness of the sky; high robustness to noise } \\
\text { (Normalized Red-Blue Ratio) }\end{array}$ & $\mathrm{NRBR}=\mathrm{R}-\mathrm{B} / \mathrm{B}+\mathrm{R}$ & [33] \\
\hline & $\mathrm{C} 1$ & $\begin{array}{c}\text { Combination of RBR, RBD, and NRBR } \\
\text { channels }\end{array}$ & RBR - RBD - NRBR & {$[17]$} \\
\hline & ARGD & $\begin{array}{c}\text { Reducing sunlight interference (Adjusted Red } \\
\text { Green Difference) }\end{array}$ & $\begin{array}{c}\mathrm{ARGD}=k \cdot \mathrm{R}-\mathrm{G} k=1.7 \text { (weight of the red } \\
\text { channel) }\end{array}$ & [22] \\
\hline & $\mathrm{C} 2$ & $\begin{array}{c}\text { Combination of RBR, ARGD, and NRBR } \\
\text { channels }\end{array}$ & RBR - RBD - ARGD & [22] \\
\hline \multirow{3}{*}{$\begin{array}{l}\text { Texture } \\
\text { feature }\end{array}$} & LR & $\begin{array}{l}\text { For distinguishing edges and contours (Local } \\
\text { Range) }\end{array}$ & Rangefilt ${ }^{1}$ & \multirow{3}{*}{ [34] } \\
\hline & STD & $\begin{array}{c}\text { Obtained from the standard deviation in each } \\
\text { neighborhood }\end{array}$ & Stdfilt $^{1}$ & \\
\hline & EM & Randomness of the image (Entropy Matrix) & Entropyfilt $^{1}$ & \\
\hline
\end{tabular}

${ }^{1}$ MATLAB function ([34]).

Different combinations of red and blue channels were proposed for cloud detection. The aim of the Red-Blue Ratio (RBR), which yields small ratios for blue skies and large ratios for clouds, is to recognize thin and opaque cloud cover and clear skies [36]. Heinle et al. [25] noted several problems related to the use of the RBR channel for detecting thick clouds and difficulties with circumsolar pixels. They therefore proposed the RBD (Red-Blue Difference) channel as an alternative. Yamashita et al. [37] performed a full revision of the blue and red channel and implemented the sky index or NRBR (Normalized Red-Blue Ratio) for separating the blue sky and clouds area. These adaptations of the RGB channels have been successfully contrasted for CD. The green channel is however often overlooked in image processing. The Adjusted Red Green Difference (ARGD) [22] was introduced to correct any possible saturation of the blue component. Linear combinations of the spectral features have been proposed in other works, such as C1 [17] and C2 [22] that are listed in Table 1.

2.3. Texture Filters. Texture filters use the gray pixel distribution (grayscale, from 0 to 255, GS matrix) and their spatial neighborhood to identify objects and regions. Texture filters divide the GS matrix into local neighbors, applying a mathematical operator: range for Local Range (LR), the Entropy Matrix (EM), and the local Standard Deviation for STD image processing [34]. Figure 1 shows an example of an LR texture-filtering process. GS is a monochromatic matrix whose elements are $M_{i, j}$. The size of the GS matrix, defined by its neighbors, is represented in Figure 1 as the $9 \times 9$ blue square. Its size is smaller throughout the GS boundary (elements represented as $m_{i, j}$ ). The filter function applies a mathematical operator in this neighborhood and the result is included in the position $(i, j)$ of the new matrix.

2.3.1. Local Range Texture Filter. The purpose of LR filtering is to make the edges and contours of an image visible. The highest value is subtracted from the smallest one within the $9 \times 9$ neighborhood, as shown in Figure 1. The function saves the result in the LR matrix.

2.3.2. EM Texture Filter. Entropy is a measure of the image texture randomness. The Entropy Matrix (EM) calculates the local entropy of all the GS neighborhoods [34]. The EM value 


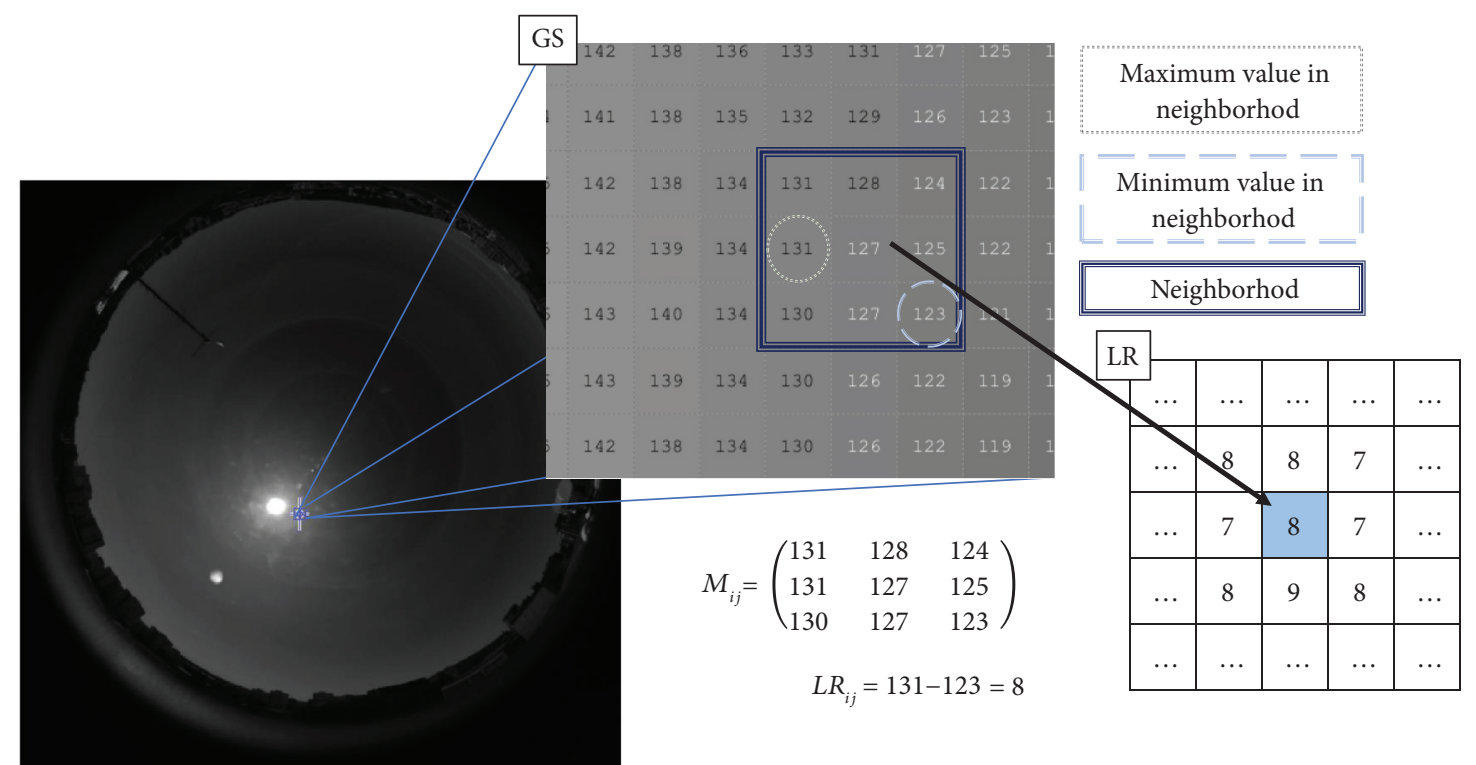

FIgURE 1: Example of LR calculation in the $9 \times 9$ neighborhood (blue area) for the element $M(i, j)=127$.

that is directly proportional to the degree of variation of a pixel with respect to its neighbors is calculated with

$$
E M_{i, j}=-\sum_{k=1}^{N} p_{k} \log _{2} p_{k}
$$

In Figure 2, an image histogram with high variations is shown. $p_{k}$ reflects the occurrence for the gray level $p$ element; $N$ is the total number of gray levels in the neighborhood.

2.3.3. Local Standard Deviation (STD) Texture Filter. The following equation is used to calculate the local Standard Deviation (STD) within each neighborhood:

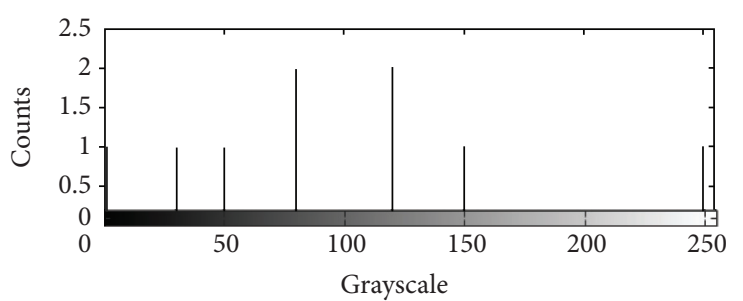

FIgURE 2: Example of the histogram for local entropy calculations. $M_{i, j}=\left(\begin{array}{ccc}1 & 50 & 80 \\ 120 & 120 & 80 \\ 250 & 150 & 30\end{array}\right)$.

$$
\operatorname{STD}_{i, j}=\sqrt{\frac{\sum_{k=1,0,-1}^{3} \sum_{l=1,0,-1}^{3}\left[M(i+k, j+l)-(1 / N)\left(\sum_{k=1,0,-1}^{3} \sum_{l=1,0,-1}^{3} M(i+k, j+l)\right)\right]^{2}}{N-1}} .
$$

$(N=9)$ is the number of elements in the neighborhood; $k, l$ varies from -1 to 1 to cover the neighborhood matrix.

\section{Experimental Data Acquisition and Processing}

As previously stated, the main objective of this work is the analysis of image-processing algorithms for CIE standard sky classification using ANN-processed sky images. The workflow is described in Figure 3 and explained in the following sections.

3.1. Experimental Data Acquisition. The experimental data used in this work were recorded at a meteorological weather station located on the roof of the Higher Polytechnic School building at Burgos University $\left(42^{\circ} 21^{\prime} 04^{\prime \prime} \mathrm{N} ; 3^{\circ} 41^{\prime} 20^{\prime \prime} \mathrm{O}\right.$; $856 \mathrm{~m}$ above mean sea level). A complete description of the meteorological facility may be found elsewhere $[1,10,38]$. The experimental equipment is shown in Figure 4. The sky luminance distribution for characterization of sky conditions according to the CIE Standard General Sky classification was measured with a commercial MS-321LR sky scanner (EKO Instruments Europe B. V. Den Haag, The Netherlands). The sky scanner was adjusted on a monthly basis for taking measurements from sunrise to the sunset. It completed a full scan in four minutes and started a new scan every 10 minutes. The first and last measurements of the day $\left(\alpha_{s} \leq 5^{\circ}\right)$ were discarded, as measurements were higher than $50 \mathrm{kcd} / \mathrm{m}^{2}$ and lower than $0.1 \mathrm{kcd} / \mathrm{m}^{2}$, following the recommended specifications of the sky scanner equipment. The 


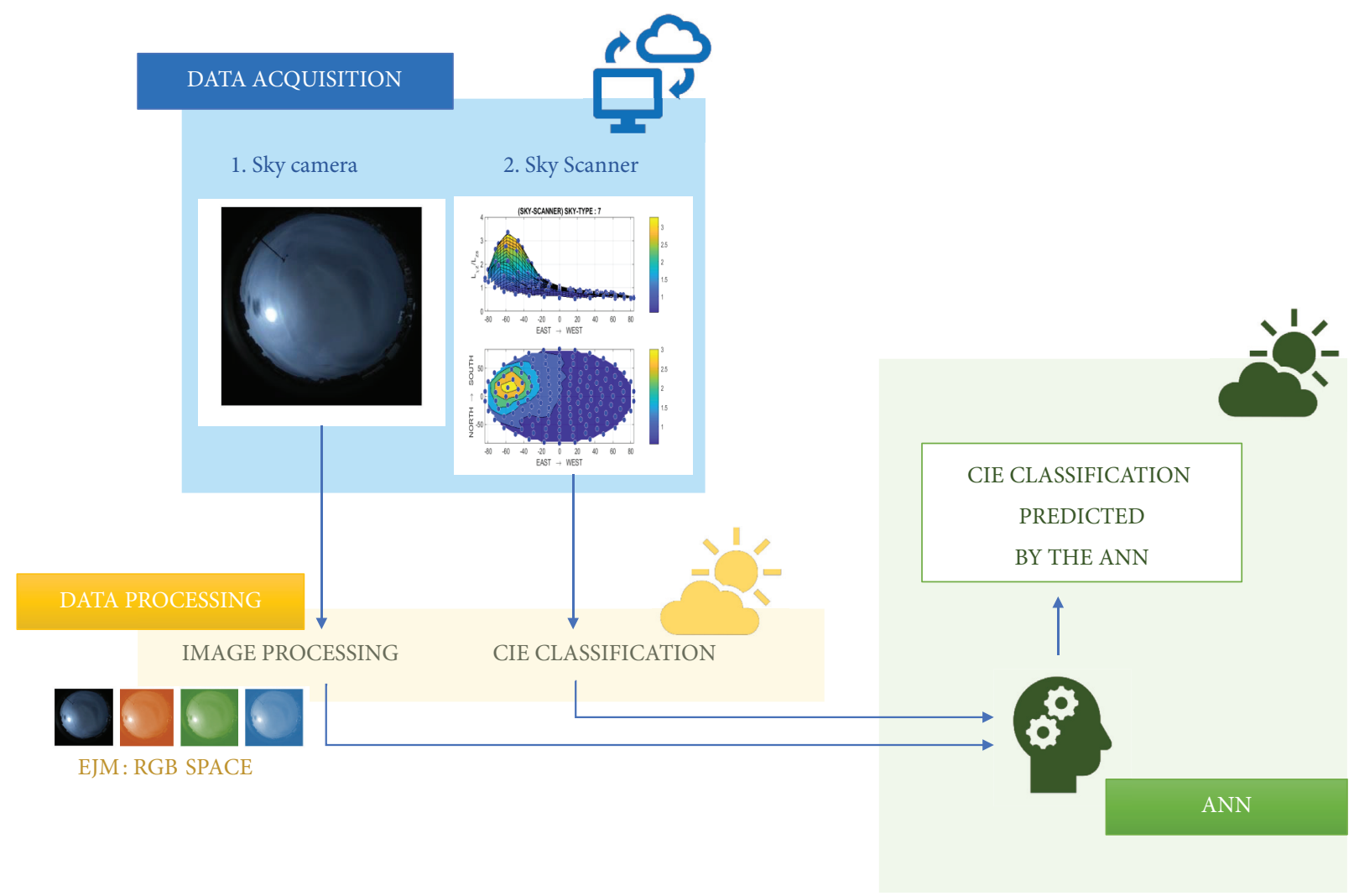

FIGURE 3: Workflow for image processing. Example with RGB space.

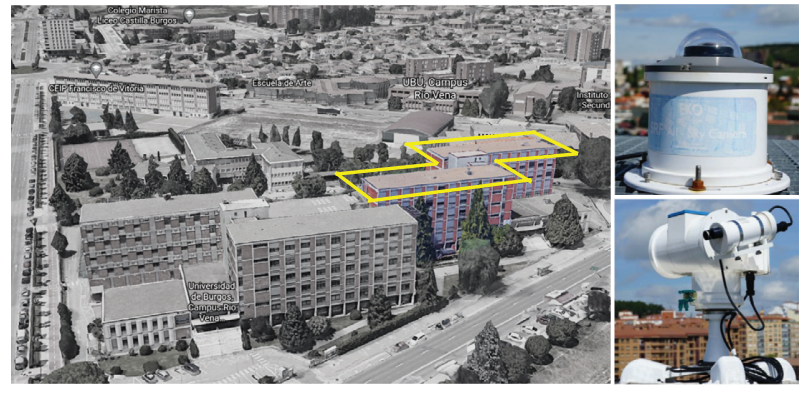

FIGURE 4: Location of the experimental equipment on the roof of the Higher Polytechnic School building at the University of Burgos with the SONA 201-D sky camera (top) and EKO MS-321LR (bottom) (source: Google Earth).

sky images were recorded every 15 seconds by a commercial SONA 201-D all sky camera day (Sieltec Canarias S L, Spain). The trigger frequency of the camera is one second and the image resolution is $1158 \times 1172$ pixels, recorded with the RGB color model (each pixel uses 8 bits, which have integer values from 0 to 255). Tables 2 and 3 show the technical specifications of the sky scanner and sky camera, respectively.

The experimental campaign took place between 1 November 2016 and 31 March 2020. The Normalized Luminance method (NL) proposed by Tregenza in 2004 [39], detailed in a previous paper [38], was used to determine the CIE standard sky types over Burgos during the experimental campaign. A total of 1,500 images were selected from the
TABLE 2: Sky scanner technical specifications.

\begin{tabular}{lc}
\hline Model & MS-321LR sky scanner \\
\hline FOV & $11^{\circ}$ \\
Luminance & 0 to $50 \mathrm{kcd} / \mathrm{m}^{2}$ \\
Radiance & 0 to $300 \mathrm{~W} / \mathrm{m}^{2}$ \\
A/D convertor & $16 \mathrm{bits}$ \\
Calibration error & $2 \%$ \\
\hline
\end{tabular}

TABle 3: Sky camera technical specifications.

\begin{tabular}{lc}
\hline Model & SONA 201-D \\
\hline Sensor & CMOS-2.3 MP \\
Vision angle & $<180^{\circ}$ (fisheye lens) \\
Operating temperature & $-40^{\circ} \mathrm{C}$ to $55^{\circ} \mathrm{C}$ \\
Image format & RAW \\
\hline
\end{tabular}

experimental dataset (more than 80,000 sky images), 100 from each CIE sky category, which were characterized by greater concordance with the CIE pattern for that category. The experimental dataset was therefore composed of one hundred sky images catalogued as CIE standard sky categories. This sky classification was used as a reference for the sky conditions.

3.2. Data Processing. The original sky images were processed using the twenty-two different channels chosen for the study and summarized in Table 1. While some of them were 
directly generated from the sky images, others had to be generated through complementary channels. In Figure 5, the results of the image-processing methods applied to images of sky conditions are classified as clear, partial, and overcast, following the CIE taxonomy. As can be observed, each filter highlights different features of the images. The circumsolar area and the nearest horizon zone present the greatest difficulties for cloud detection. In Figure 5, it can be seen that the RGB image is sensitive to the circumsolar region and is capable of detecting the solar corona. However, in the RGB image, no differences can be appreciated in dark-homogenous sky conditions. The appearance of direct day beam can be a source of errors. Although the blue channel saturated the circumsolar region, both the red and the green channels showed greater sensitivity at detecting cloudy areas. In contrast, the horizon was captured by the Y, D, L, RAS, V, STD, and EM methods, and LR mainly defined the contours. Unlike most of the other channels, the RGB model had difficulty with the directional homogeneity of the images for the detection of overcast sky conditions. The family of RAS methods (RAS, Y, D, and L) appeared to show similar levels of accuracy under all sky conditions, their main differences being near the circumsolar area.

3.3. Image Compression. The high resolution of the original sky images $(1158 \times 1172$ pixels $)$ requires their compression to reduce the dimension of the dataset, improving data storage and subsequent image processing. In this study, the original sky images were compressed to $110 \times 110$ pixels in each channel. Figure 6 shows the result of the image compression procedure to $0.89 \%$, which facilitates ANN tuning with no loss of efficiency.

\section{ANN for CIE Standard Sky Classification}

Artificial Neural Networks (ANNs) are frequently used in meteorology science: CIE and cloud classification [40, 41], solar irradiance and wind speed forecasting [42-47], atmospheric pollution distribution [48, 49], and rainfall $[50,51]$. ANN classification models serve to classify input information into certain categories or targets. A Supervised Machine Learning (SML) neural network is required for CIE standard sky classification where the sky types are previously known. The model works efficiently when the prediction matches the target. Modelled on the biological concept of neurons, ANN is a very powerful technique for classification problems. Figure 7 shows a conventional ANN structure, which consists of an input layer, a set of several hidden layers, and an output layer.

The information from the neurons of the input layer $\left(X_{i}^{0}\right)$ crosses the hidden layers (one in this work), following unidirectional connections, to the output layer that has one neuron $\left(X_{i}^{2^{\prime}}\right)$ per target. Each processing center or neuron is adjusted to the other neurons through an interactive process, using (3). The Scaled Conjugate Gradient method (SCG) [52] was used to fit the weights (weighting matrix, $W^{n}$ ) for each iteration.

$$
X_{i}^{n}=W^{n} X^{n-1}+B,
$$

where $W^{n}$ is the weighting matrix, $X^{n-1}$ are the input variables, and $B$ is the bias. The neuron generates the output, $X_{i}^{n^{\prime}}$, through the activation function, $f\left(X_{i}^{n}\right)$, given by the hyperbolic tangent sigmoid transfer function in this study, as shown in [13]

$$
X_{i}^{n^{\prime}}=f\left(X_{i}^{n}\right)=\frac{2}{1+\exp \left(-2 \cdot X_{i}^{n}\right)}-1 .
$$

Supervised Machine Learning requires three datasets: training, validation, and test datasets. The training group is used to determine the weighted matrix and the bias in an iterative process. The training is over when the results of the performance of the resulting model, calculated using the validation set, reach the desired quality. The test data group is used to calculate the performance of the model. Random dataset division is crucial to achieve a reliable performance. A conventional training dataset is randomly selected and consists of $70 \%$ of the total data, while the validation set and the test set each represent $15 \%$, respectively.

The design of the ANN is adapted to the database and the process is simulated. There is no standardized procedure for establishing the most effective number of neurons and hidden layers [42], so experimentation or tuning is needed. In this study, several trials were performed in which the number of neurons (1-100) was varied, searching for the best accuracy, Acc, of the ANN, given by

$$
\mathrm{Acc}=\frac{\mathrm{TP}+\mathrm{TN}}{\mathrm{TP}+\mathrm{TN}+\mathrm{FP}+\mathrm{FN}},
$$

where TP and TN are the correct predictions of the ANN (true positives and true negatives) and FP and FN are the incorrect predictions (false positives and false negatives). Accuracy is rated by the number of correct predictions over the total number of predictions. The neural network structure (number of neurons in the hidden layer) was selected on the basis of highest accuracy. After several trials, the number of hidden layers was fixed at one.

\section{Results}

In Figure 8, the improved accuracy of the ANN models that used the sky images as their input is shown. Each image had previously been processed by each of the twenty-one imageprocessing methods summarized in Table 1 , with respect to the RGB space, defined as $\Delta$ (Acc) and shown in

$$
\Delta(\mathrm{Acc})=\frac{(\text { Acc }(\text { channel } x)-\mathrm{Acc}(\mathrm{RGB} \text { space }))}{\operatorname{Acc}(\mathrm{RGB} \text { space })} \cdot 100,
$$

where Acc (channel $x$ ) and Acc (RGB space) are the accuracy obtained when the input of the ANN is the set of sky images processed by each method $x$ ( $x=$ each image-processing method summarized in Table 1) and RGB space, respectively. The accuracy of each ANN and the number of neurons in its hidden layer are shown in Table 4. 


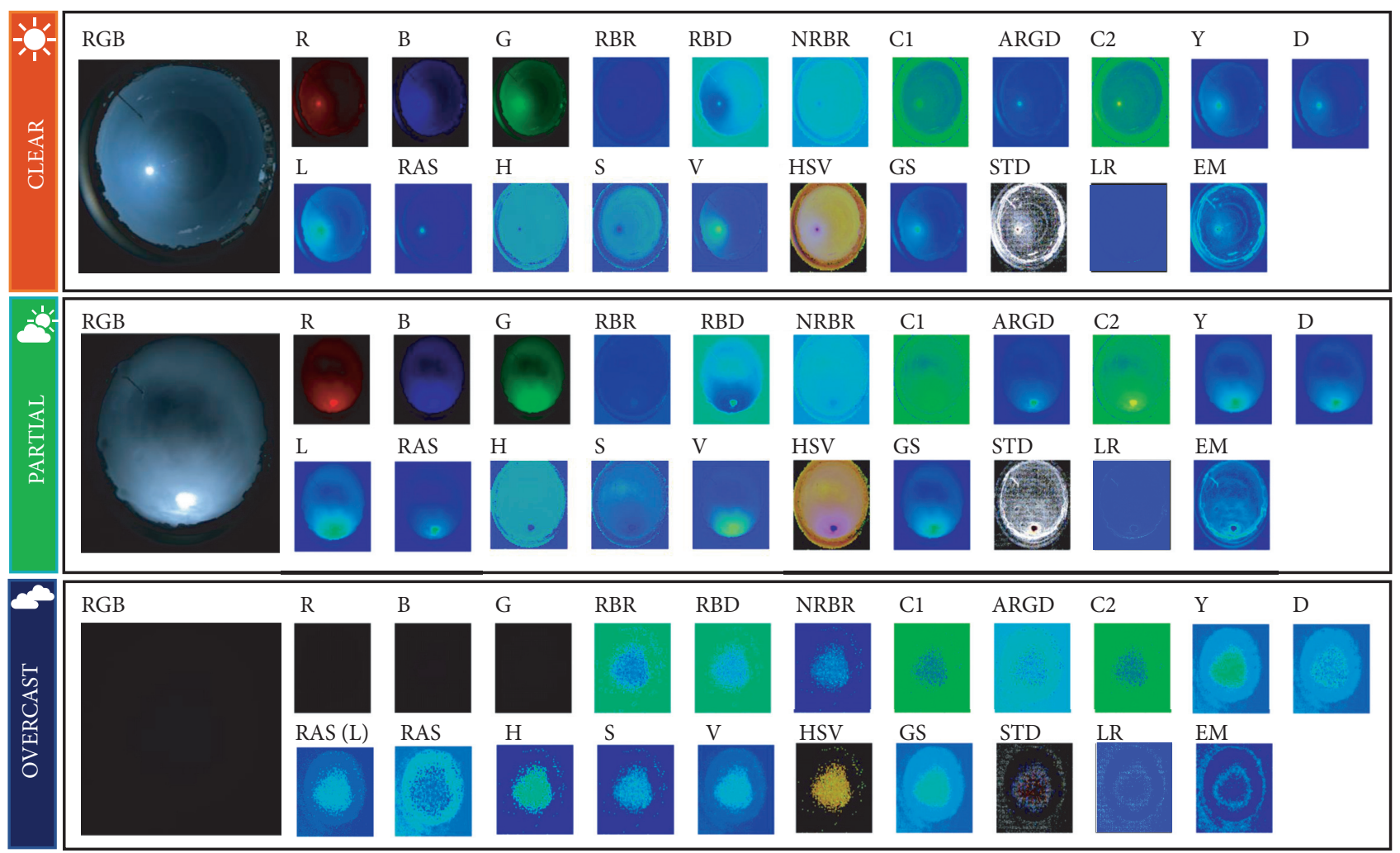

FIGURE 5: Results of the image-processing methods applied to clear, partial, and overcast CIE standard sky types.

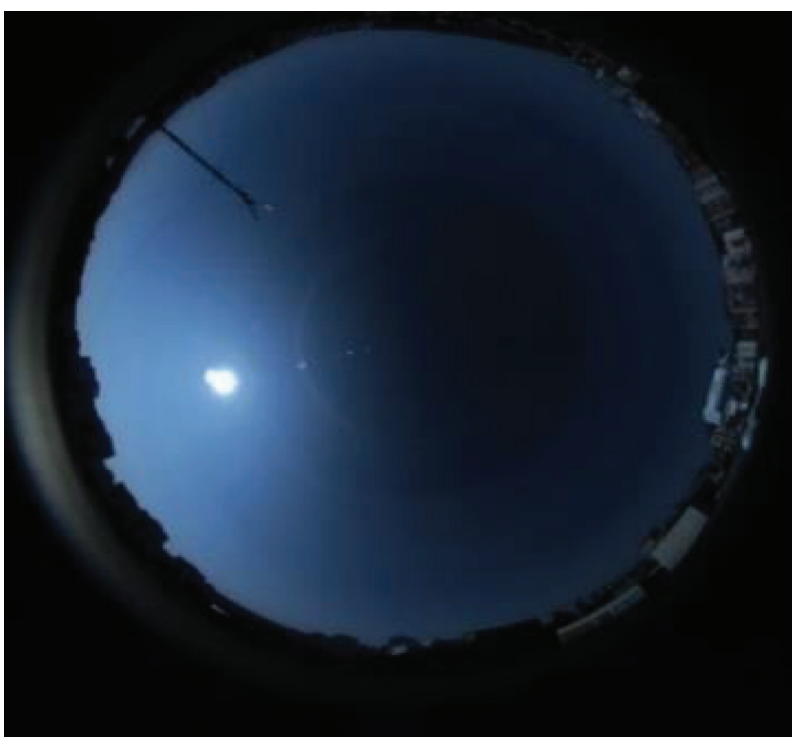

(a)

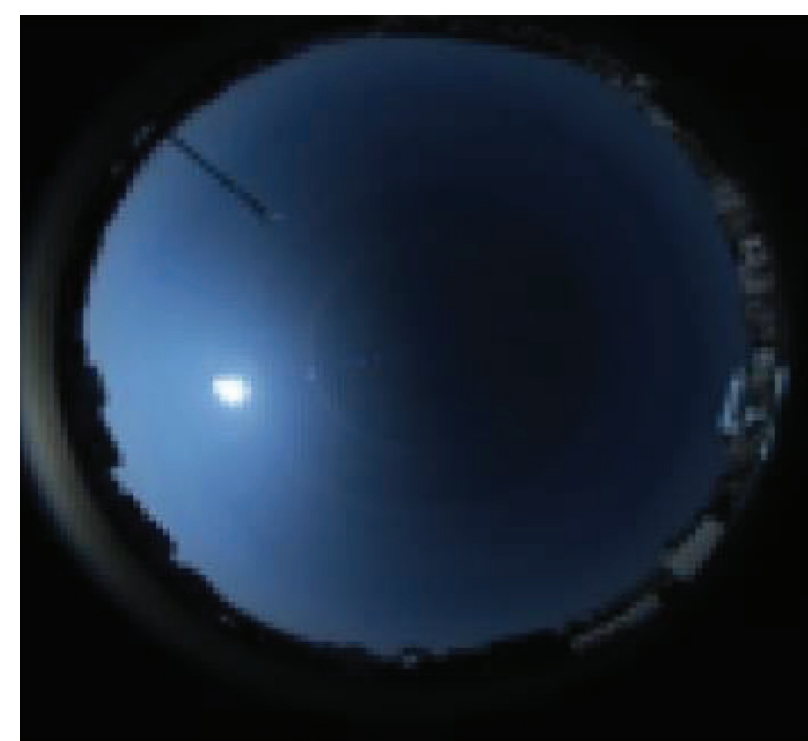

(b)

FIGURE 6: Result of the image compression procedure. (a) Actual sky image. (b) Compressed sky image.

As can be seen in Figure 8, HSV is better color space than RGB for CIE standard sky classification using images, with a small improvement in the accuracy $(0.66 \%)$ with respect to RGB image processing. The GS color space and the RGB space were equally accurate. The use of the R, G, and $B$ monochromatic channels also improved the accuracy of the ANN for CIE standard sky classification, the $\mathrm{G}$ channel being the most suitable for this task. The accuracy of the ANN fitted using the individual channels, $\mathrm{H}$ and $\mathrm{S}$, worsened over the RGB color space, while the $\mathrm{V}$ channel significantly improved ANN accuracy. 


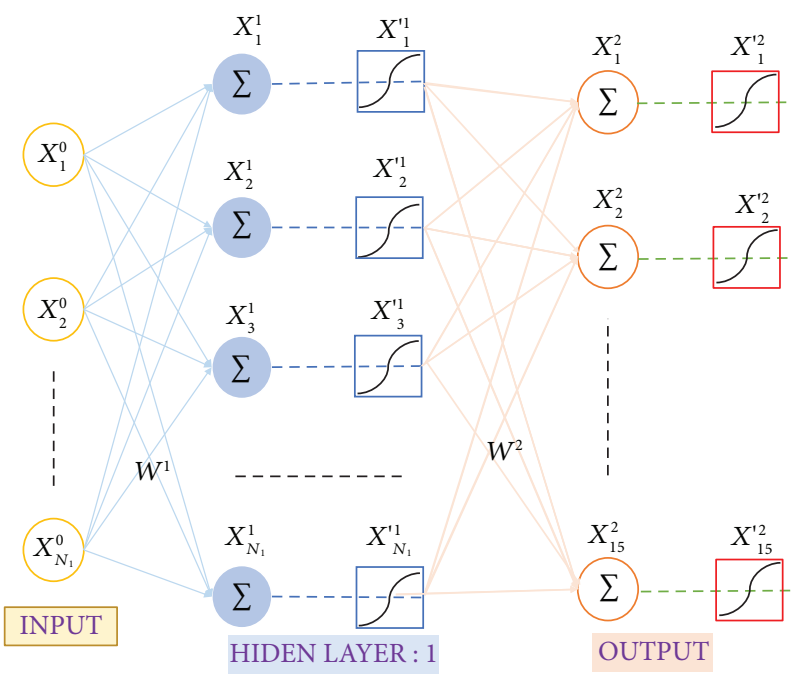

FIgURE 7: ANN system architecture.

$-17.34$

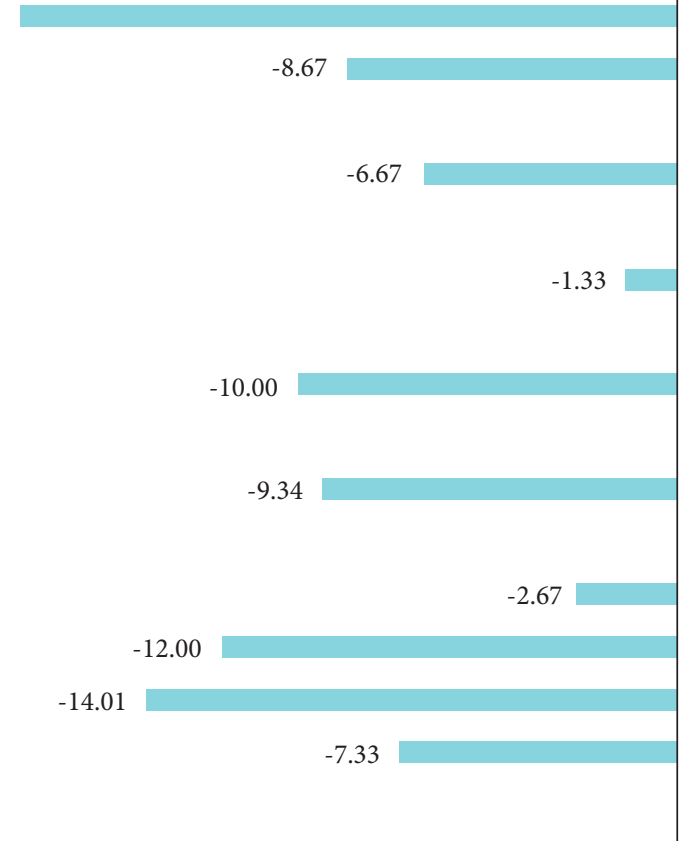

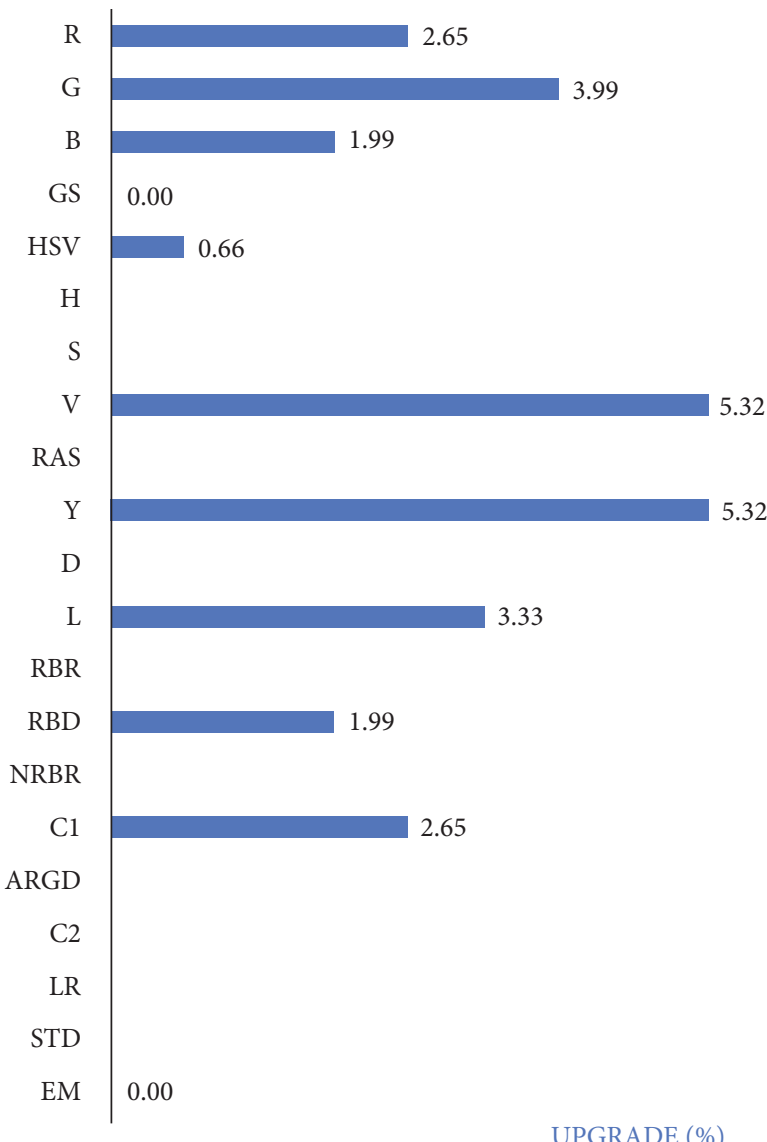

UPGRADE (\%)

Figure 8: Improvement in ANN accuracy, $\Delta$ (Acc), for CIE standard sky classification, using the results of each image-processing method as input, as summarized in Table 1, over ANN accuracy obtained with the original RGB images as input.

In the spectral feature category, the RAS processing method worsened the sky classification accuracy of the ANN. However, channels Y and L showed better behavior for sky classification, although they used more neurons in the hidden layer. Among the rest of spectral feature channels, only RBD and C1 significantly improved ANN accuracy. With regard to the texture filters, EM showed little or no advantages over the use of the RGB color space and the other two filters, LR and STD, impaired the accuracy of the resulting neural network. The number of neurons in the 
TABLE 4: Accuracy and structure (number of neurons in the hidden layer) of the selected ANN for each image-processing method.

\begin{tabular}{lcc}
\hline Method & Acc $(\%)$ & Number of neurons \\
\hline RGB & 66.67 & 84 \\
R & 68.44 & 66 \\
G & 69.33 & 58 \\
B & 68 & 36 \\
GS & 66.67 & 58 \\
HSV & 67.11 & 40 \\
H & 55.11 & 23 \\
S & 60.89 & 27 \\
V & 70.22 & 58 \\
RAS & 62.22 & 25 \\
Y & 70.22 & 95 \\
D & 65.78 & 21 \\
L & 68.89 & 74 \\
RBR & 60 & 45 \\
RBD & 68 & 98 \\
NRBR & 60.44 & 27 \\
C1 & 68.44 & 73 \\
ARGD & 64.89 & 17 \\
C2 & 58.67 & 68 \\
LR & 57.33 & 39 \\
STD & 61.78 & 52 \\
EM & 66.67 & 58 \\
\hline
\end{tabular}

hidden layer, shown in Table 4, never increased the accuracy of the ANN, as can be seen from the use of image-processing methods $\mathrm{Y}$ and $\mathrm{V}$.

Figure 8 shows the results of each ANN classifying the skies into the fifteen CIE standard sky categories. A simpler classification into three categories (clear, overcast, and partial conditions) is often sufficient for many applications, such as luminous efficacy calculations [53] and lighting design in buildings [54]. The fitted results of the ANN sky classification for three categories are shown in Figure 9 and Table 5.

For CIE standard sky classification into three sky categories, lower differences in accuracy can be seen and only the G, the B, and the GS monochromatic channels and the spectral features L and C1 improved ANN accuracy. In all these classification cases, the number of neurons in the hidden layer was lower.

The accuracy index was used to group the goodness of fit of the ANN in all categories, although the fitted quality in each individual category was not processed. A confusion matrix analysis is shown in Figures 10-13. In a confusion matrix, when the Supervised Machine Learning algorithm prediction and the target match each other (TP or TN result), the corresponding diagonal boxes of the matrix are colored. When there are no matches between the prediction and the target value (FP and FN), the other boxes of the confusion matrix are filled in. The best image-processing method will have the highest number of colored boxes around the diagonal line of the matrix. The figures below represent the confusion matrices corresponding to the 15 types of CIE standard skies.

Figure 10 shows the confusion matrix of the ANNcalculated RGB-CIE sky classification for the test set (15\% of the total dataset). It can be seen that the RGB-CIE classification with machine learning misclassified cloudy and partial skies: few matches are visible in the boxes along the diagonal line. In Figure 10, the CIE standard sky classification into three categories (clear, partial, and overcast sky conditions) is also presented. Those cases classified outside the corresponding category were designated as critical, i.e., clear skies classified as either partial or overcast or vice versa.

The same information is shown in Figure 11 for the color space CIE standard sky classification, corresponding to the other color space processing methods under analysis. The red, the green, and the blue channels showed a similar behavior to the RGB color space. The red channel adequately classified CIE standard sky types 7 to 15 , in other words, all clear skies and some partial sky types. The HSV color space showed a similar performance in all categories, in contrast to the RGB color space, in which the classification of clear sky types may be highlighted. Hue and saturation channels introduced too much noise, but the value channel showed good performance.

In Figure 12, the confusion matrices are shown for the spectral feature image-processing methods-CIE standard sky classification. The RBR and NRBR spectral features introduced noise, but the resultant combination, C1, reduced misclassification, improving the traditional RGB color space. It therefore appears to be an adequate alternative image-processing method for CIE standard sky classification using sky camera images. The RAS channel theoretically removed atmospheric scattering, but the confusion matrix never reflected a better performance than the RGB color space. The confusion matrix has demonstrated that it cannot distinguish the CIE sky types 1, 3, and 5. The RAS method also introduced too much noise in cloudy-to-partial sky types.

Finally, the confusion matrices are shown in Figure 13 for texture filter processing methods-CIE standard sky classification with ANN. As can be seen, all texture channels performed well, especially the EM channel, while LR largely failed for CIE standard sky classifications partial and overcast.

A detailed study for the CIE standard sky classification into three categories is presented in Figure 14, where the confusion matrices presented in Figures 10-13 were divided into four submatrices: overcast (CIE standard sky types 1 to 5 ), partial (CIE standard sky types 6 to 10), clear (CIE standard sky types 1 to 5 ), and critical that refers to cases classified out of category. The red line indicates the RGB result, taken as a baseline for accuracy improvements, $\Delta$ (Acc). Some of the image-processing methods for classifying certain sky categories are highlighted in Figure 14. RBD, D, and B showed the best performance for the detection of overcast skies, increasing the performance of each respective ANN. G, S, and GS achieved better results for the detection of partial skies and clear skies were also in the same category in which the conventional RGB color space achieved its best performance.

Some channels highlighted certain sky types but drastically failed to classify other types. The blue channel saturated in clear skies, to such a point that its performance was 


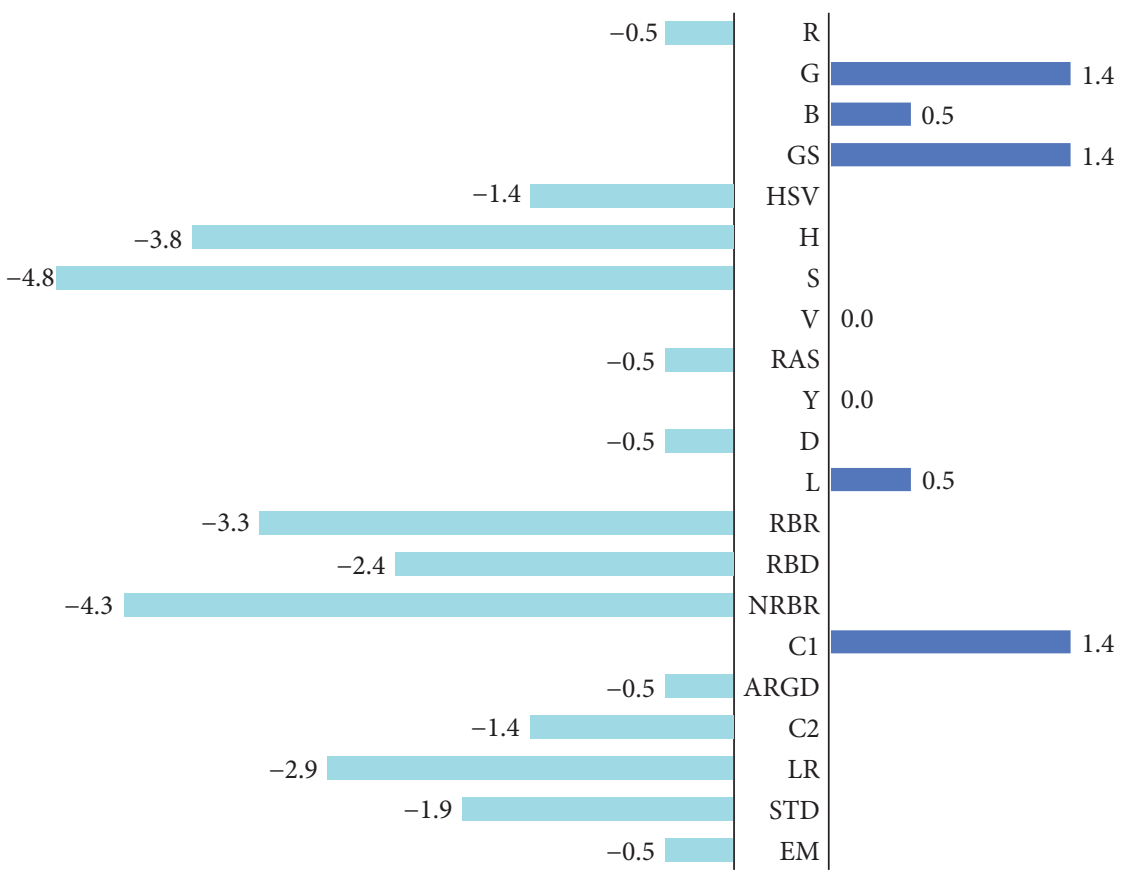

UPGRADE (\%)

FIGURE 9: Improvement in ANN accuracy, $\Delta$ (Acc), for CIE standard sky classification in three sky categories: overcast, partial, and clear conditions, using as input the image processed by each image-processing method summarized in Table 1, over ANN accuracy obtained with the RGB images as input.

TABLE 5: Accuracy and structure (number of neurons in the hidden layer) of the ANN selected for each image-processing method.

\begin{tabular}{lcc}
\hline Method & Acc $(\%)$ & Number of neurons \\
\hline RGB & 93.33 & 71 \\
R & 92.89 & 48 \\
G & 94.67 & 52 \\
B & 93.78 & 52 \\
GS & 94.67 & 52 \\
HSV & 92 & 11 \\
H & 89.78 & 23 \\
S & 88.89 & 73 \\
V & 93.33 & 15 \\
RAS & 92.89 & 90 \\
Y & 93.78 & 21 \\
D & 92.89 & 15 \\
L & 93.78 & 15 \\
RBR & 90.22 & 23 \\
RBD & 91.11 & 43 \\
NRBR & 89.33 & 23 \\
C1 & 94.67 & 18 \\
ARGD & 92.89 & 19 \\
C2 & 92 & 41 \\
LR & 90.67 & 81 \\
STD & 91.56 & 7 \\
EM & 92.89 & 68 \\
\hline & &
\end{tabular}

almost the worst for clear skies detection. This behavior was also noted for the $\mathrm{D}$ channel.

Unfortunately, no image preprocessing method drastically improved the RGB classification in the three

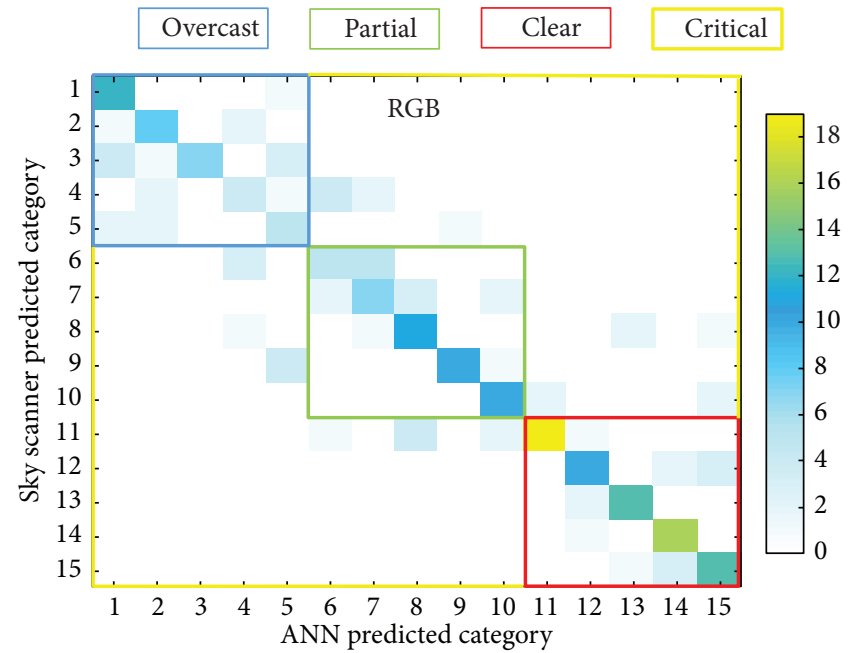

FIgURE 10: Confusion matrix for ANN-calculated RGB-CIE sky classification into fifteen categories and into three categories: overcast (CIE standard sky types 1 to 5), partial (CIE standard sky types 6 to 10), and clear (CIE standard sky types 1 to 5). Critical refers to cases classified out of category. The color scale shows the number of coincidences in each category.

subcategories (clear, partial, and overcast conditions). However, Y, green, red, RBD, V, and EM processing methods were prominent in one or two categories and their results were acceptable in all other categories, as shown in Table 6. 


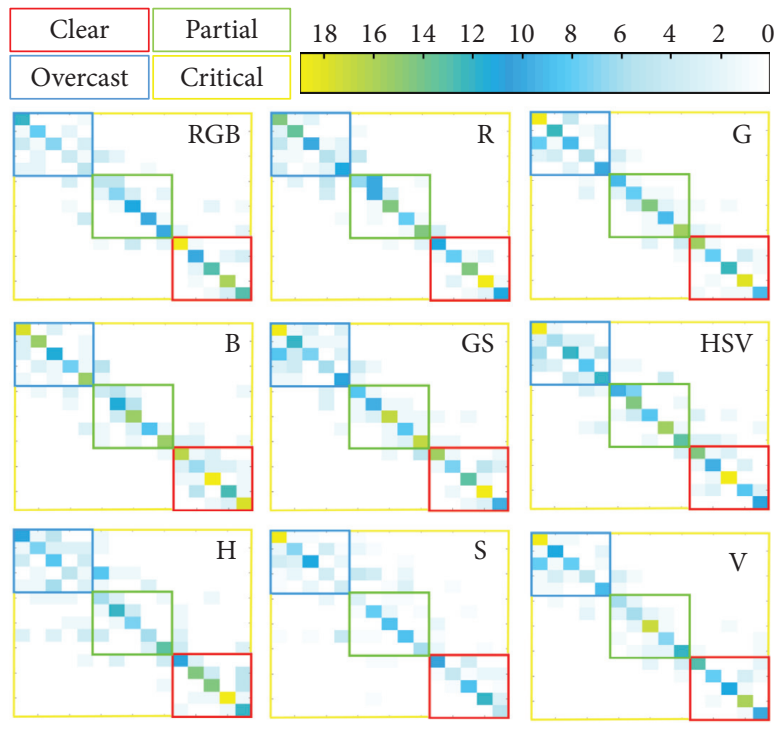

FIGURE 11: Confusion matrices color space image-processing methods-CIE standard sky classification.

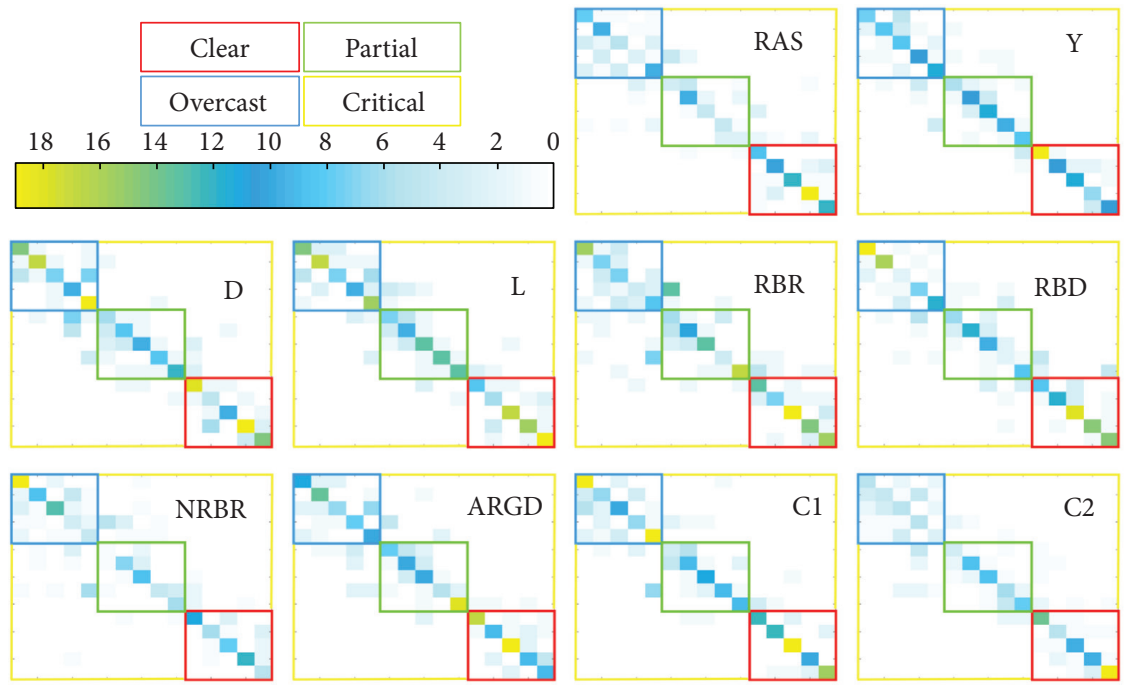

FIGURE 12: Confusion matrices spectral feature image-processing methods-CIE standard sky classification.

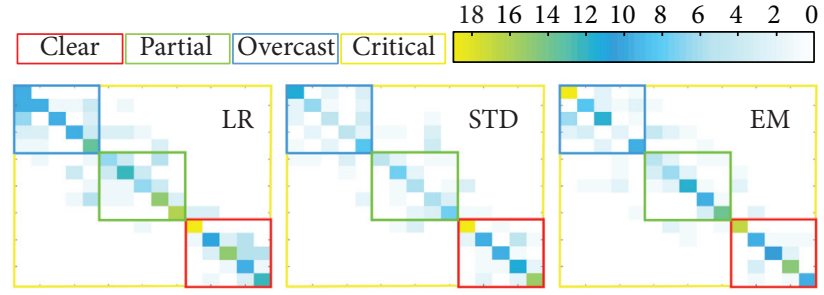

FIGURE 13: Confusion matrices for texture filter image-processing methods-CIE standard sky classification. 

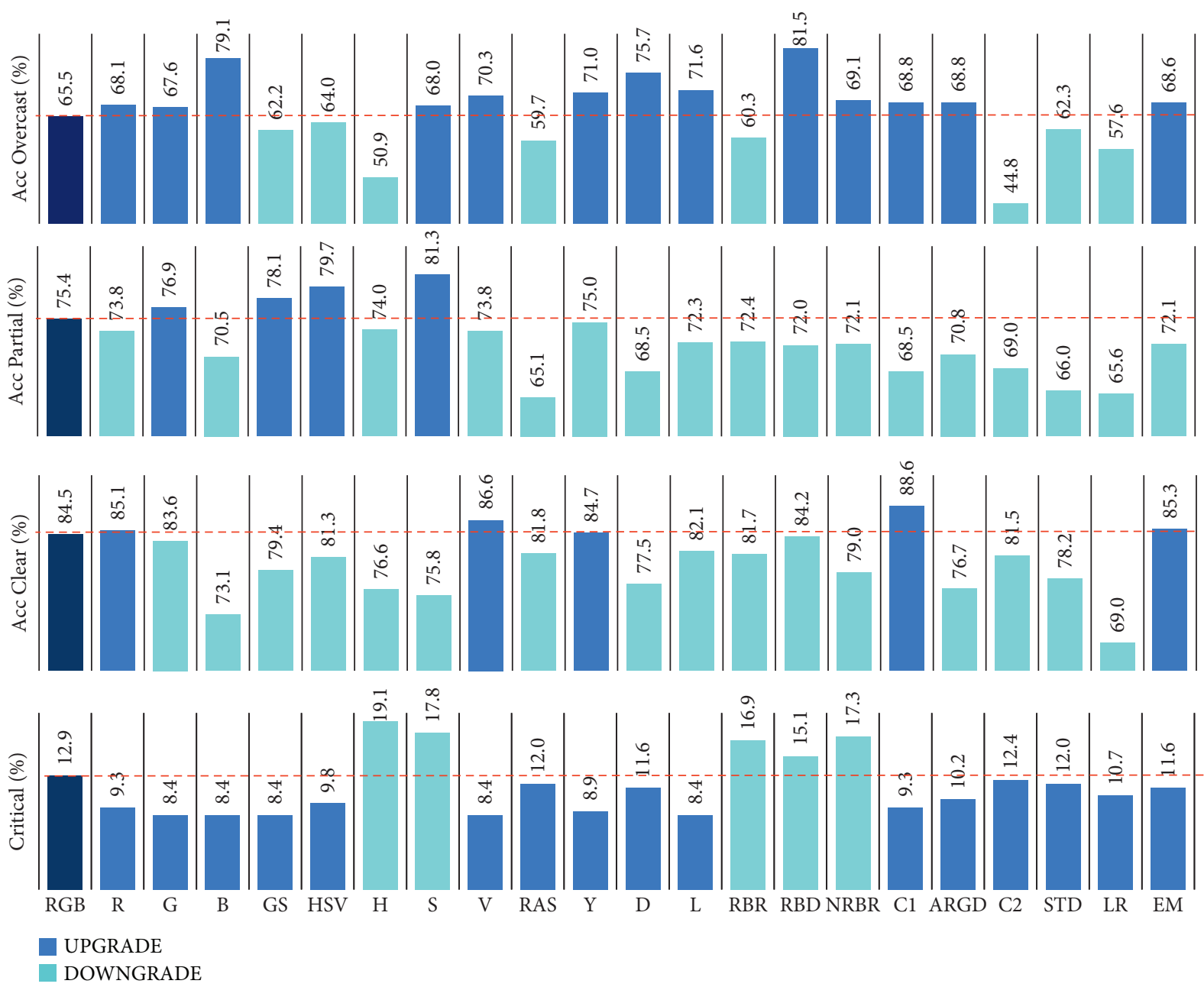

FIGURE 14: Acc for the CIE standard sky classification through sky images and ANN, using the different image-processing methods recorded in Table 1.

TABLE 6: Summary of the results.

\begin{tabular}{lccc}
\hline CIE categories & One category & Two categories & Three categories \\
\hline Clear & R, C1, Y, EM, and V & \\
Partial & G, S, HSV, and GS & \\
$\begin{array}{l}\text { Overcast } \\
\text { Critical }\end{array}$ & R, G, B, RBD, NRBR, C1, ARGD, Y, D, L, S, V, and EM & R, G, C1, Y, S, V, and EM & R, G, Y, and V, EM \\
\hline
\end{tabular}

Almost all the image-processing methods reduced critical mistakes or misclassification, which should as far as possible be avoided. Following this criterion, RBR, RBD, NRBR, ARGD, H, and $S$ were discarded as preprocessing image methods for ANN sky classification of sky images.

\section{Conclusions}

Sky classification and cloud detection from sky images and machine learning can be largely improved through preliminary image processing, reducing errors in classification and simplifying algorithms. In this study, 22 sky imageprocessing methods have been reviewed, including the three most common categories, color spaces, spectral features, and texture filters. The CIE standard sky classification has been selected to determine the characteristics of the sky, as it is recognized as representative of the atmospheric conditions. A very extensive unbiased dataset has been used, including 1,500 sky images and their corresponding CIE classification, calculated through the Normalized Luminance method from sky luminance distribution data. The Artificial Neural Network (ANN) was the selected machine learning algorithm.

As a first conclusion, digital cameras equipped with fisheye lens can be used as alternatives to sky scanner devices for ANN-assisted CIE standard sky classification. The accuracy of the classification algorithm can be improved with adequate preliminary image processing that highlights the sky image information and optimizes the algorithmic structure. 
HSV was a better color space than RGB, as were the monochromatic channels $\mathrm{R}, \mathrm{G}$, and $\mathrm{B}$, for classifying the skies on the basis of the images into the fifteen CIE standard sky types. Only the $\mathrm{V}$ individual channel of HSV worked better than both HSV and RGB. Spectral feature channels Y and $\mathrm{L}$ showed better behavior for sky classification than the RGB color space, but they used more neurons in the hidden layer. Among the rest of the spectral feature channels, only RBD and C1 significantly improved ANN accuracy. Texture filters added no significant advantages over the RGB color space.

For CIE standard sky classification as clear, partial, and overcast conditions, RGB appeared to be the best imageprocessing method and only the monochromatic channels $\mathrm{G}$ and B, GS, and the composed spectral feature C1 improved the accuracy of the RGB color space. No improvement in ANN performance was therefore noted with the use of extra channels.

In contrast to previous studies [14] which have their weakest accuracy in cloudy conditions, several channels have worked successfully, improving the accuracy of the machine learning algorithm by $10 \%$ over the RGB color space for cloudy skies. These channels were B, R, S, V, ARGD, RBD, C1, C2, Y, STD, and EM.

RGB and its primary channels, R, G, and B, were not good enough for dark cloudy conditions, due to imageprocessing information losses. While traditional cloud detection has usually omitted the $G$ channel, both the $G$ and the $B$ channels have been shown to be equally effective. In contrast, the B channel tended to saturate on clear sky conditions.

The confusion matrices highlighted that the ANN failed to distinguish CIE sky types 1,3 , and 5 .

The main conclusion is that the use of a specific imageprocessing method could improve the accuracy of an ANN algorithm, depending on the information required from the image for the classification problem. Future work will focus on the classification of skies according to the CIE standard using neural networks specifically designed for the classification of images such as convolutional neural networks.

\section{Data Availability}

The neural network database used to support the findings of this study has been deposited in the Institutional Repository of University of Burgos (https://riubu.ubu.es/).

\section{Conflicts of Interest}

The authors declare that they have no conflicts of interest.

\section{Authors' Contributions}

D. Granados-López participated in investigation, methodology, software, and visualization; A. García-Rodríguez and S. García-Rodríguez took part in investigation, methodology, formal analysis, and validation; A. Suárez-García contributed to methodology and software; M. DiezMediavilla was responsible for conceptualization, original draft preparation, and supervision as well as funding acquisition and project administration; C. Alonso Tristán played a role in conceptualization, original draft preparation, and supervision (writing, reviewing, and editing).

\section{Acknowledgments}

The authors gratefully acknowledge the financial support provided by the Regional Government of Castilla y León under the "Support Program for Recognized Research Groups of Public Universities of Castilla y León" (BU021G19) and the Spanish Ministry 595 of Science and Innovation under the $\mathrm{I}+\mathrm{D}+\mathrm{i}$ state program "Challenges Research Projects” (Ref. RTI2018-098900-B-I00). Diego Granados López expresses his thanks for economic support from the Junta de Castilla-León (PIRTU Program, ORDEN EDU/556/2019).

\section{References}

[1] A. Suárez-García, M. Díez-Mediavilla, D. Granados-López, A. González-Peña, and C. Alonso-Tristán, "Benchmarking of meteorological indices for sky cloudiness classification," Solar Energy, vol. 195, pp. 499-513, 2020.

[2] R. Kittler, S. Darula, and R. Perez, A Set of Standard Skies Characterizing Daylight Conditions for Computer and Energy Conscious Design, Final Report, Slovak Academy of Science, Slovakia, 1998.

[3] Y. Uetani, S. Aydinli, A. Joukoff, and J. D. Kendrick, Spatial Distribution of Daylight-CIE Standard General Sky, ISOCIE, Geneva, Vienna, 2004.

[4] D. H. W Li, S. Li, W. Chen, and S. Lou, "Analysis of point daylight factor (PDF) average daylight factor (ADF) and vertical daylight factor (VDF) under various unobstructed CIE standard skies," IOP Conference Series: Materials Science and Engineering, vol. 556, no. 1, Article ID 12044, 2019.

[5] D. H. W. Li, S. Lou, J. C. Lam, and R. H. T. Wu, "Determining solar irradiance on inclined planes from classified CIE (International Commission on Illumination) standard skies," Energy, vol. 101, pp. 462-470, 2016.

[6] H. Xiao, Q. Kang, J. Zhao, and Y.-s. Xiao, "A dynamic sky recognition method for use in energy efficient lighting design based on CIE standard general skies," Building and Environment, vol. 45, no. 5, pp. 1319-1328, 2010.

[7] I. García, M. de Blas, and J. L. Torres, "The sky characterization according to the CIE Standard General Sky: comparative analysis of three classification methods," Solar Energy, vol. 196, pp. 468-483, 2020.

[8] "Guide to recommended practice of daylight measurement, CIE publication 108, central bureau of the CIE, Vienna, Austria 1994, softbound, 54 pp., \$59 (members)/\$88 (nonmembers)," Color Research and Application, vol. 20, no. 1, p. 80, 1995.

[9] D. H. W. Li and S. Lou, "Review of solar irradiance and daylight illuminance modeling and sky classification," Renewable Energy, vol. 126, pp. 445-453, 2018.

[10] D. Granados-López, D. Suárez-GarcÍa, D. Díez-Mediavilla, and C. Alonso-Tristán, "Feature selection for CIE standard sky classification,” Solar Energy, vol. 218, pp. 95-107, 2021.

[11] S. Lou, D. H. W. Li, and J. C. Lam, "CIE Standard Sky classification by accessible climatic indices," Renewable Energy, vol. 113, pp. 347-356, 2017. 
[12] A. Taravat, F. del Frate, C. Cornaro, and S. Vergari, "Neural networks and support vector machine algorithms for automatic cloud classification of whole-sky ground-based images," IEEE Geoscience and Remote Sensing Letters, vol. 12, no. 3, pp. 666-670, 2014.

[13] T. Kazanasmaz, M. Günaydin, and S. Binol, "Artificial neural networks to predict daylight illuminance in office buildings," Building and Environment, vol. 44, no. 8, pp. 1751-1757, 2009.

[14] D. H. W. Li, H. L. Tang, E. W. M. Lee, and T. Muneer, "Classification of CIE standard skies using probabilistic neural networks," International Journal of Climatology, vol. 30, no. 2, pp. 305-315, 2010.

[15] D. H. W. Li, H. L. Tang, S. L. Wong, E. K. W. Tsang, G. H. W. Cheung, and T. N. T. Lam, "Skies classification using artificial neural networks (ANN) techniques," in Proceedings of the 6th International Conference on Indoor Air Quality, Ventilation and Energy Conservation in Buildings: Sustainable Built Environment, IAQVEC, Sendai Japan, October 2007.

[16] J. Alonso-Montesinos and F. J. Batlles, "The use of a sky camera for solar radiation estimation based on digital image processing," Energy, vol. 90, pp. 377-386, 2015.

[17] R. Chauvin, J. Nou, S. Thil, A. Traoré, and S. Grieu, "Cloud detection methodology based on a sky-imaging system," Energy Procedia, vol. 69, pp. 1970-1980, 2015.

[18] H.-Y. Cheng and C.-L. Lin, "Cloud detection in all-sky images via multi-scale neighborhood features and multiple supervised learning techniques," Atmospheric Measurement Techniques, vol. 10, no. 1, pp. 199-208, 2017.

[19] M. B. Piderit, C. Cauwerts, and M. Diaz, "Definition of the CIE standard skies and application of high dynamic range imaging technique to characterize the spatial distribution of daylight in Chile," Revista de la construcción, vol. 13, no. 2, pp. 22-30, 2014.

[20] D. Granados-López, I. Suárez-García, M. Díez-Mediavilla, and C. Alonso-Tristán, "Evaluation of two procedures for selecting the CIE standard sky type using high dynamic range images," in Proceedings of the ISES Solar World Congress 2019, SWC 2019 and IEA SHC International Conference on Solar Heating and Cooling for Buildings and Industry 2019, SHC, Santiago, Chile, January 2019.

[21] X. Wan and J. Du, "Cloud classification for ground-based sky image using random forest," The International Archives of the Photogrammetry, Remote Sensing and Spatial Information Sciences, vol. XLIII-B3-2020, pp. 835-842, 2020.

[22] X. Li, Z. Lu, Q. Zhou, and Z. Xu, "A cloud detection algorithm with reduction of sunlight interference in ground-based sky images," Atmosphere, vol. 10, no. 11, p. 640, 2019.

[23] P. Seuntiens, M. Van Boven, and D. Sekulovski, "Effect of skylight configuration and sky type on the daylight impression of a room," WIT Transactions on Ecology and the Environment, vol. 165, pp. 53-61, 2012.

[24] J. Yang, Q. Min, W. Lu, Y. Ma, W. Yao, and T. Lu, “An RGB channel operation for removal of the difference of atmospheric scattering and its application on total sky cloud detection," Atmospheric Measurement Techniques, vol. 10, no. 3, pp. 1191-1201, 2017.

[25] A. Heinle, A. Macke, and A. Srivastav, "Automatic cloud classification of whole sky images," Atmospheric Measurement Techniques, vol. 3, no. 3, pp. 557-567, 2010.

[26] M. P. Souza-Echer, E. B. Pereira, L. S. Bins, and M. A. R. Andrade, "A simple method for the assessment of the cloud cover state in high-latitude regions by a ground-based digital camera," Journal of Atmospheric and Oceanic Technology, vol. 23, no. 3, pp. 437-447, 2006.
[27] Q. Luo, Y. Meng, L. Liu, X. Zhao, and Z. Zhou, "Cloud classification of ground-based infrared images combining manifold and texture features," Atmospheric Measurement Techniques, vol. 11, no. 9, pp. 5351-5361, 2018.

[28] P. K. Mall, P. K. Singh, and D. Yadav, "GLCM based feature extraction and medical $\mathrm{X}$-ray image classification using machine learning techniques," in Proceedings of the 2019 IEEE Conference on Information and Communication Technology, IEEE, Allahabad, India, December 2019.

[29] L. Wei and D. Hong-ying, "Real-time road congestion detection based on image texture analysis," Procedia engineering, vol. 137, pp. 196-201, 2016.

[30] P. Maillard, "Spectral-textural image classification in a semiarid environment," in Proceedings of the ISPRS Commission VII Symposium 'Remote Sensing: From Pixels to Processes', Enschede Citeseer, The Netherlands, May 2006.

[31] S. Tuominen and A. Pekkarinen, "Performance of different spectral and textural aerial photograph features in multisource forest inventory," Remote Sensing of Environment, vol. 94, no. 2, pp. 256-268, 2005.

[32] X. Zhaoa and H. Zheng, "Cloud detection of gray photographs by cloud region detection and optimal region grow threshold," in Proceedings of the 2nd International Conference on Intelligent Computing and Cognitive Informatics (ICICCI 2015), Singapore, 2015.

[33] J. Yang, Q. Min, W. Lu et al., "An automated cloud detection method based on the green channel of total-sky visible images," Atmospheric Measurement Techniques, vol. 8, no. 11, pp. 4671-4679, 2015.

[34] "Image processing toolbox for use with MATLAB, user's Guide version 5. 2004," The MathWorks, Inc.vol. 5, 2004, https://es.mathworks.com/help/pdf_doc/images/images_ref. pdf.

[35] K. F. Hussain and H. A. Sayed, "Sky detection using K-HSV descriptor," Journal of the Institute of Industrial Applications Engineers, vol. 2, no. 1, pp. 1-5, 2014.

[36] T. Koehler, R. Johnson, and J. Shields, "Status of the whole sky imager database," in Proceedings of the Cloud Impacts on DOD Operations and Systems, pp. 77-80, Department of Defense, El Segundo, CA, USA, 1991.

[37] M. Yamashita, M. Yoshimura, and T. Nakashizuka, "Cloud cover estimation using multitemporal hemisphere imageries. International Archives of Photogrammetry," Remote Sensing and Spatial Information, vol. 35, pp. 826-829, 2004.

[38] A. Suárez-García, D. Granados-López, D. González-Peña, M. Díez-Mediavilla, and C. Alonso-Tristán, "Seasonal caracterization of CIE standard sky types above Burgos, northwestern Spain," Solar Energy, vol. 169, pp. 24-33, 2018.

[39] P. Tregenza, "Analysing sky luminance scans to obtain frequency distributions of CIE Standard General Skies," Lighting Research and Technology, vol. 36, no. 4, pp. 271-279, 2004.

[40] D. H. W. Li, T.C. Chau, and K.W. Wan, "A review of the CIE general sky classification approaches," Renewable and Sustainable Energy Reviews, vol. 31, pp. 563-574, 2014.

[41] B. Bin Tian, M. A. Shaikh, M. R. Azimi-Sadjadi, T. H. V. Haar, and D. L. Reinke, "A study of cloud classification with neural networks using spectral and textural features," IEEE Transactions on Neural Networks, vol. 10, no. 1, pp. 138-151, 1999.

[42] F. J. L. Lima, F. R. Martins, E. B. Pereira, E. Lorenz, and D. Heinemann, "Forecast for surface solar irradiance at the Brazilian Northeastern region using NWP model and artificial neural networks," Renewable Energy, vol. 87, pp. 807-818, 2016. 
[43] H. Calik, N. Ak, and I. Guney, "Artificial NARX neural network model of wind speed: case of Istanbul-Avcilar," Journal of Electrical Engineering and Technology, vol. 16, no. 5, pp. 2553-2560, 2021.

[44] Y. Chen, Y. Wang, Z. Dong et al., "2-D regional short-term wind speed forecast based on CNN-LSTM deep learning model," Energy Conversion and Management, vol. 244, Article ID 114451, 2021.

[45] G. Goudarzi, P. K. Hopke, and M. Yazdani, "Forecasting PM2.5 concentration using artificial neural network and its health effects in Ahvaz, Iran," Chemosphere, vol. 283, 2021.

[46] Y. Kassem, H. Gökçekuş, and W. Janbein, "Predictive model and assessment of the potential for wind and solar power in Rayak region, Lebanon," Modeling Earth Systems and Environment, vol. 7, no. 3, pp. 1475-1502, 2021.

[47] B. Shboul, I. Alarfi, S. Michailos, and D. Ingham, "A new ANN model for hourly solar radiation and wind speed prediction: a case study over the north \& south of the Arabian Peninsula," Sustainable Energy Technologies and Assessments, vol. 46, Article ID 101248, 2021.

[48] S. Gao, Z. Bai, S. Liang et al., "Simulation of surface ozone over Hebei province, China using Kolmogorov-Zurbenko and artificial neural network (KZ-ANN) combined model," Atmospheric Environment, vol. 261, Article ID 118599, 2021.

[49] G. Gualtieri, S. D. Lonardo, F. Carotenuto et al., "The role of emissions and meteorology in driving $\mathrm{CO}_{2}$ concentrations in urban areas," Environmental Science and Pollution Research, vol. 28, no. 23, pp. 29908-29918, 2021.

[50] M. Ghamariadyan and M. A. Imteaz, "Monthly rainfall forecasting using temperature and climate indices through a hybrid method in Queensland, Australia," Journal of Hydrometeorology, vol. 22, no. 5, pp. 1259-1273, 2021.

[51] P. Wellyantama and S. Soekirno, "Temperature, pressure, relative humidity and rainfall sensors early error detection system for automatic weather station (AWS) with artificial neural network (ANN) backpropagation," in Proceedings of the 10th International Conference on Theoretical and Applied Physics (ICTAP), Lombok, Indonesia, 2020.

[52] M. F. Møller, "A scaled conjugate gradient algorithm for fast supervised learning," Neural Networks, vol. 6, no. 4, pp. 525-533, 1993.

[53] M. I. Dieste-Velasco, M. Díez-Mediavilla, D. GranadosLópez, D. González-Peña, and C. Alonso-Tristán, "Performance of global luminous efficacy models and proposal of a new model for daylighting in Burgos, Spain," Renewable Energy, vol. 133, pp. 1000-1010, 2019.

[54] H. Kambezidis, T. Oikonomou, and D. Zevgolis, "Daylight climatology in the Athens urban environment: guidance for building designers," Lighting Research and Technology, vol. 34, no. 4, pp. 297-309, 2002. 